\title{
Targeted therapy in lung cancer-ASCO 2019 update
}

\author{
Romana E. Wass (D) - David Lang · Bernd Lamprecht · Michael Studnicka
}

Received: 31 July 2019 / Accepted: 17 October 2019 / Published online: 6 November 2019

(C) The Author(s) 2019

\begin{abstract}
Summary New targeted therapies for patients with non small cell lung cancer were presented at this year's ASCO. EGFR exon 20 insertions might soon be treatable with TAK-788, which showed an objective response rate (ORR) of between 25 and $56 \%$ with a disease control rate (DCR) between 67 and $100 \%$ depending on the presence or absence of brain metastases at baseline. Capmatinib and tepotinib showed durable responses in MET exon 14 mutations as presented in the phase II GEOMETRY trial and the VISION trial. The median duration of response (DOR) was 9.7 months with a median progression free survival (PFS) of 5.42 months in pretreated patients and 11.14 months respective 9.6 months in those receiving capmatinib in the frontline setting. Tepotinib showed similar results with a median DOR of 12.4 months and a DCR of $66.7 \%$. For RET-fusion lung cancer the well tolerated RET inhibitor BLU-667 has already been granted breakthrough therapy designation. Among previously treated patients an ORR of $58 \%$ and a DCR
\end{abstract}

\section{R. E. Wass, M.D., Ph.D. $(\bowtie)$ · D. Lang, M.D. .}

\section{B. Lamprecht, M.D.}

Department of Pulmonary Medicine,

Kepler-University-Hospital GmbH, 4020 Linz, Austria

romana@wass.at

\section{Lang, M.D.}

david.lang@kepleruniklinikum.at

B. Lamprecht, M.D.

bernd.lamprecht@kepleruniklinikum.at

R. E. Wass, M.D., Ph.D. · D. Lang, M.D. · B. Lamprecht, M.D. Faculty of Medicine, Johannes-Kepler-University, 4020 Linz, Austria

R. E. Wass, M.D., Ph.D. · M. Studnicka, M.D.

Department of Pneumology, Paracelsus Medical University, 5020 Salzburg, Austria

m.studnicka@salk.at of $96 \%$ was reported. The median duration of response had not yet been reached, with many patients continuing to respond for longer than 24 months. For the large and hard-to-treat group of patients with $K R A S$-mutated lung cancer, early data on the covalent irreversible inhibitor of the KRASG12C-mutant kinase AMG 510 were presented. In pretreated patients AMG 510 achieved a $50 \%$ response rate, with a duration of treatment from 7.3 to 27.4 weeks. Furthermore, AMG 510 has been safe and well tolerated at dose levels tested in 35 patients in dose exploration.

Keywords Non small cell lung cancer · Hard-to target alterations · Rare mutations · EGFR exon 20 - KRAS

\section{Background}

In the last few years, treatment options for non-small cell lung cancer (NSCLC) have rapidly evolved. In addition to the advent of checkpoint inhibitors that revolutionized the therapeutic landscape for a large fraction of NSCLC patients, genomic profiling triggered the identification of an increasing number of potentially druggable driver mutations.

At the 2019 meeting of the American Society of Clinical Oncology (ASCO), major advances regarding NSCLC genetics and druggable targets were presented-some showing encouraging findings for patients with rare mutations such as RET (REarranged during transfection), $c$-MET (mesenchymal epithelial transition factor), ROS-1 (ROS proto-oncogene 1 , receptor tyrosine kinase) or hard-to-target alterations such as Epidermal growth factor receptor (EGFR) exon 20 insertions and Kirsten rat sarcoma (KRAS). Even though these therapies are not yet ready for use in daily clinical practice, they are raising hope that a larger proportion of NSCLC patients may soon be 
able to benefit from such molecular targeted therapies.

\section{EGFR: uncommon mutations - potential targeted treatment for exon $\mathbf{2 0}$ insertion}

Exon 20 is the third most common alteration in EGFR, following the activating mutations in exon 19 and exon 21, which are successfully targeted by tyrosine kinase inhibitors (TKIs) such as osimertinib, dacomitinib, afatinib, gefitinib and erlotinib. Despite the high efficacy and good tolerability of these drugs in patients with activating mutations, they are largely ineffective for patients with insertions in exon 20 .

Jänne et al. presented results of TAK-788-a TKI, which potently inhibits EGFR exon 20 mutations [1]. TAK-788 was examined in a multicohort phase I/II trial for patients with advanced non-small cell lung cancer (NSCLC). In a dose-escalation phase of the study, the maximum tolerated dose was determined to be $160 \mathrm{mg}$ daily. Twenty-eight patients from the trial who were treated with $160 \mathrm{mg}$ were analyzed for the presentation at the ASCO meeting.

Forty-three percent of patients in this analysis had brain metastases at baseline. The median age of the patients was 62 years, and the most common ECOG status was 1 (79\%). The median number of prior systemic cancer treatments was 3 , including prior checkpoint inhibitors $(61 \%)$ and EGFR- or HER2-directed TKIs (18\%).

The confirmed objective response rate (ORR) was $25 \%$ in patients with baseline brain metastases and a disease control rate (DCR) of $67 \%$. In those without brain metastases, the confirmed ORR was 56\% and the DCR was $100 \%$. The median progression-free survival (PFS) was 7.3 months across all patients in the analysis and was 3.7 months and 8.1 months for those with and without brain metastases, respectively. Grade $\geq 3$ treatment-related adverse events (AEs) occurred in $63 \%$ of patients treated at the $160 \mathrm{mg}$ dose. The most common treatment-related grade $\geq 3$ AEs were diarrhea $(18 \%)$, nausea $(6 \%)$, increased lipase (6\%), stomatitis (4\%), and increased amylase (4\%).

Another therapy in development, poziotinib, has also shown potential for patients with EGFR exon 20 insertions, according to findings presented at the IASLC 19th World Conference on Lung Cancer. This therapy induced a confirmed ORR of $43 \%$ among evaluable patients with EGFR exon 20 mutant NSCLC [2].

Further studies exploring both treatments are ongoing. The EXCLAIM expansion cohort is currently examining TAK-788 at $160 \mathrm{mg}$ daily in 91 patients (NCT02716116) and a phase II study exploring poziotinib continues to recruit participants (NCT03066206).
MET: exon 14 skip-mutation-two new targeted treatments are on the way

MET exon 14 skipping mutations have been identified in approximately 3-4\% of NSCLC cases and are associated with a poor response to currently available therapies. At the 2019 ASCO Annual Meeting updated data for the two MET inhibitors capmatinib and tepotinib for patients with MET-exon-14-altered lung cancer were presented. Both agents showed deep and durable responses both in previously treated and treatment-naïve patients.

In the first-line setting, capmatinib showed an ORR by independent review (IR) of $67.9 \%$ and a DCR of $96.4 \%$; in pretreated patients, the ORR by IR was $40.6 \%$ and the DCR was $78.3 \%$ according to data from the phase II GEOMETRY trial [3], which was presented by J. Wolf. The GEOMETRY trial examined capmatinib in different cohorts; cohort 4 contained pretreated patients with $M E T$ exon 14 alterations in the secondor third-line setting $(n=69)$, while cohort $5 \mathrm{~b}$ included treatment-naive patients $(n=28)$.

The median duration of response (DOR) by IR was 9.7 months in pretreated patients and 11.14 months in those receiving capmatinib in the frontline setting. The median progression-free survival was 5.42 months in the pretreated group and 9.6 months for those treated in the frontline setting.

Approximately half of the patients with brain metastases at baseline $(15.9 \%$ in cohort $4,10.7 \%$ in cohort 5b) experienced an intracranial response with capmatinib with an especially high DCR of $92.3 \%$.

Grade 3 treatment-related AEs occurred in $31.1 \%$ of patients and a grade 4 event was seen in $4.5 \%$ of patients. The most common treatment-related AE of any grade was peripheral edema $(41.6 \%)$, nausea $(33.2 \%)$, increased blood creatinine $(19.5 \%)$, and vomiting (18.9\%).

Tepotinib-the second selective MET inhibitor that was presented-showed similar results in the phase II VISION trial [4]. Across all lines of treatment, the ORR by IR in liquid biopsy-identified MET exon14-positive tumors was $50 \%$. The median DOR was 12.4 months and the DCR was $66.7 \%$. For those identified by tissue biopsy, the ORR was $45.1 \%$ and the median DOR was 15.7 months with a DCR of $72.5 \%$.

By line of therapy, the ORR by IR was $58.8 \%$ in the first-line and $53.3 \%$ in the second-line in the liquid biopsy-identified patients. In the second-line or higher, the ORR by IR was still $45.5 \%$.

The median duration of response across arms was 14.3 months. The most common treatmentrelated AEs were peripheral edema $(48.3 \%)$, nausea $(23.0 \%)$, diarrhea $(20.7 \%)$, and blood creatinine increase $(12.6 \%)$.

These promising results should further encourage testing for this rare driver mutation as more targeted treatment options may soon be available in clinical practice. 
RET-fusion: selective RET inhibitors show durable responses in previously treated patients

Until recently, there was a lack of effective targeted treatment options for patients with advanced RETfusion-positive lung cancer. Multikinase inhibitors like cabozantinib, vandetanib, lenvatinib with several other targets in addition to RET showed limited efficacy but high toxicity $[5,6]$.

Gainor et al. presented preliminary efficacy data with the RET inhibitor BLU-667 [7]. BLU-667 was explored in a dose-escalation study that resulted in a maximum tolerated dose of $400 \mathrm{mg}$ per day. In the expansion study, 120 patients with RET-positive advanced NSCLC have already been treated. The median age of patients in the study was 60 years, and $62 \%$ had an ECOG performance status of 1 or 2 . Brain metastases were present for $40 \%$ of patients at baseline and patients had received a median of two prior therapies, including chemotherapy $(77 \%)$ or a checkpoint inhibitor (39\%).

Among previously treated patients with RET-fusion-positive NSCLC an ORR of $58 \%$ and a DCR of $96 \%$ was reported. The median duration of response had not yet been reached, with many patients continuing to respond for longer than 24 months. Responses were seen regardless of RET fusion partner, CNS involvement, and prior frontline therapy.

The most common treatment-related grade $\geq 3$ toxicities were neutropenia (13\%), hypertension $(10 \%)$, anemia $(4 \%)$, fatigue $(3 \%)$, leukopenia $(3 \%)$, and increased CPK (3\%), constipation (2\%), AST increase $(2 \%)$, and ALT increase (2\%). Seven percent of patients discontinued treatment due to treatment-related toxicity.

A similar magnitude of benefit has also been reported with another RET inhibitor, LOXO-292 [8]. Recently both agents have been granted breakthrough therapy designations from the FDA for use in previously treated patients with advanced RET-fusion-positive lung cancer.

KRAS - is there light on the horizon for a hard-totarget alteration?

KRAS mutations comprise approximately $30 \%$ of all NSCLCs and are known to be difficult to target, which is why there is still no effective targeted therapy available for this large group of patients.

This years' ASCO raised some hope in that field, when early data on the covalent irreversible inhibitor of the KRASG12C-mutant kinase AMG 510 were presented. The KRASG12C inhibitor was evaluated in 35 previously treated patients with locally advanced or metastatic NSCLC $(n=14)$, colorectal cancer $(n=19)$, and appendix cancer $(n=2)$. All patients had received at least two lines of prior systemic therapy. AMG 510 achieved a $50 \%$ response rate in patients with
KRASG12C-positive NSCLC, according to results from a phase I study presented by Fakih et al. [9].

Among the NSCLC responders, the duration of treatment ranged from 7.3 to 27.4 weeks and from 8.4 to 25.1 weeks among patients with NSCLC who had stable disease. Most common AEs included diarrhea, decreased appetite, and nausea. There were no dose-limiting toxicities, grade 4 AEs, or serious AEs. AMG 510 has been safe and well tolerated at dose levels tested in 35 patients in dose exploration. Further studies are needed to see if AMG 510 might be a feasible treatment option for KRAS mutant NSCLC patients.

\section{Conclusion}

ASCO 2019 NSCLC highlights were dominated by a multitude of novel targeted therapy options. Next to the presented data on substances targeting relatively rare alterations like EGFR exon 20, MET exon 14 or $R E T$, especially the findings reported for KRASG12C seem promising and may pose valuable treatment options for a number of NSCLC patients in the near future. With that ongoing expansion of targeted therapy options for NSCLC, an even greater emphasis should be put upon molecular characterization of NSCLC including a broader application of NGS.

\section{Take home message}

Encouraging results for rare and hard-to-treat molecular alterations in NSCLC were presented at this year's ASCO. TAK-788 and poziotinib might be future targeted treatment options for patients with exon 20 insertion EGFR-mutated lung cancer, capmatinib and tepotinib showed deep and durable responses in patients with MET exon 14 skip mutations and the RET inhibitor BLU667 has even been granted breakthrough therapy designation from the FDA because of promising data presented by J. Gainor at this year's meeting. Even though AMG 510 is not yet ready for use in clinical practice, the results are very encouraging for the large and hard-totreat group of patients with KRAS-mutated lung cancer, who have an especially poor prognosis.

Funding Open access funding provided by Johannes Kepler University Linz.

Conflict of interest R.E. Wass, D. Lang, B. Lamprecht, and M. Studnicka declare that they have no competing interests.

Open Access This article is distributed under the terms of the Creative Commons Attribution 4.0 International License (http://creativecommons.org/licenses/by/4.0/), which permits unrestricted use, distribution, and reproduction in any medium, provided you give appropriate credit to the original author(s) and the source, provide a link to the Creative Commons license, and indicate if changes were made. 


\section{References}

1. Jänne PA, Neal JW, Cambridge DR, et al. Antitumor activity of TAK-788 in NSCLC with EGFR exon 20 insertions. J Clin Oncol. 2019;37(suppl):abstr9007.

2. Heymach J, Negrao M, Robichaux J, et al. A phase II trial of poziotinib in EGFR and HER2 exon 20 mutant non-small cell lung cancer (NSCLC). Presented at: The IASLC 19th World Conference on Lung Cancer; 23.09.-26.09.; Toronto. 2018. p. AbstractOA02.06..

3. Wolf J, Seto T, Han J-H, et al. Capmatinib (INC280) in MET $\Delta$ ex14-mutated advanced non-small cell lung cancer (NSCLC): efficacy data from the phase II GEOMETRY mono-1 study. J Clin Oncol. 2019;37(suppl 15):abstr 9004. https://doi.org/10.1200/JCO.2019.37.15_suppl.9004.

4. Pail PK, Vellion R, Cortot AB, et al. Phase II study of tepotinib in NSCLC patients with METex14 mutations. J Clin Oncol. 2019;37(suppl 15):abstr 9005.

5. Drilon A, Rekhtman N, Arcila M, et al. Cabozantinib in patients with advanced RET-rearranged non-small-cell lung cancer: an open-label, single-centre, phase 2 , singlearm trial. Lancet Oncol. 2016;17(12):1653-60.

6. GautschiO, MiliaJ, Filleron T, etal. TargetingRET in patients with RET-rearrangendlung cancers: results from the global, multicenterRETregistry. JClin Oncol. 2017;35(13):1403-10.

7. Gainor JF, Lee DH, Curigliano G, et al. Clinical activity and tolerability of BLU-667, a highly potent and selective RET inhibitor, in patients (pts) with advanced RET-fusion+ nonsmall cell lung cancer (NSCLC). J Clin Oncol. 2019;37(suppl 15):abstr 9008.

8. Oxnard G, Subbiah V, Park K, et al. Clinical activity of LOXO292, a highly selective RET inhibitor, in patients with RET fusion+non-small cell lung cancer. Presented at: The IASLC 19th World Conference on Lung Cancer; 23.09.-26.09.; Toronto. 2018. p. AbstractOA12.07.

9. Fakih M, Price TJ, O'Neil B, Price TJ, et al. Phase 1 study evaluating the safety, tolerability, pharmacokinetics (PK), and efficacy of AMG 510, a novel small molecule KRASG12C inhibitor, in advanced solid tumors. J Clin Oncol. 2019;37(suppl 15):abstr 3003.

Publisher's Note Springer Nature remains neutral with regard to jurisdictional claims in published maps and institutional affiliations.

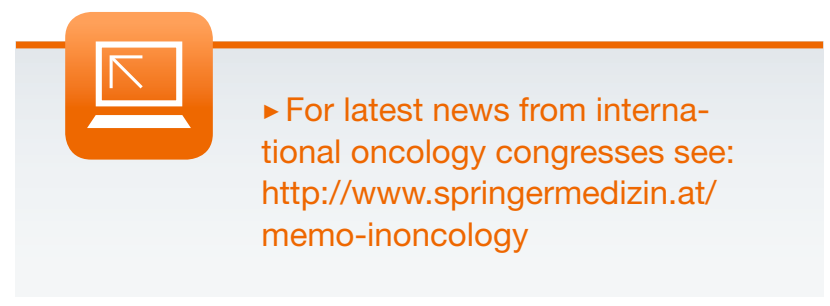

